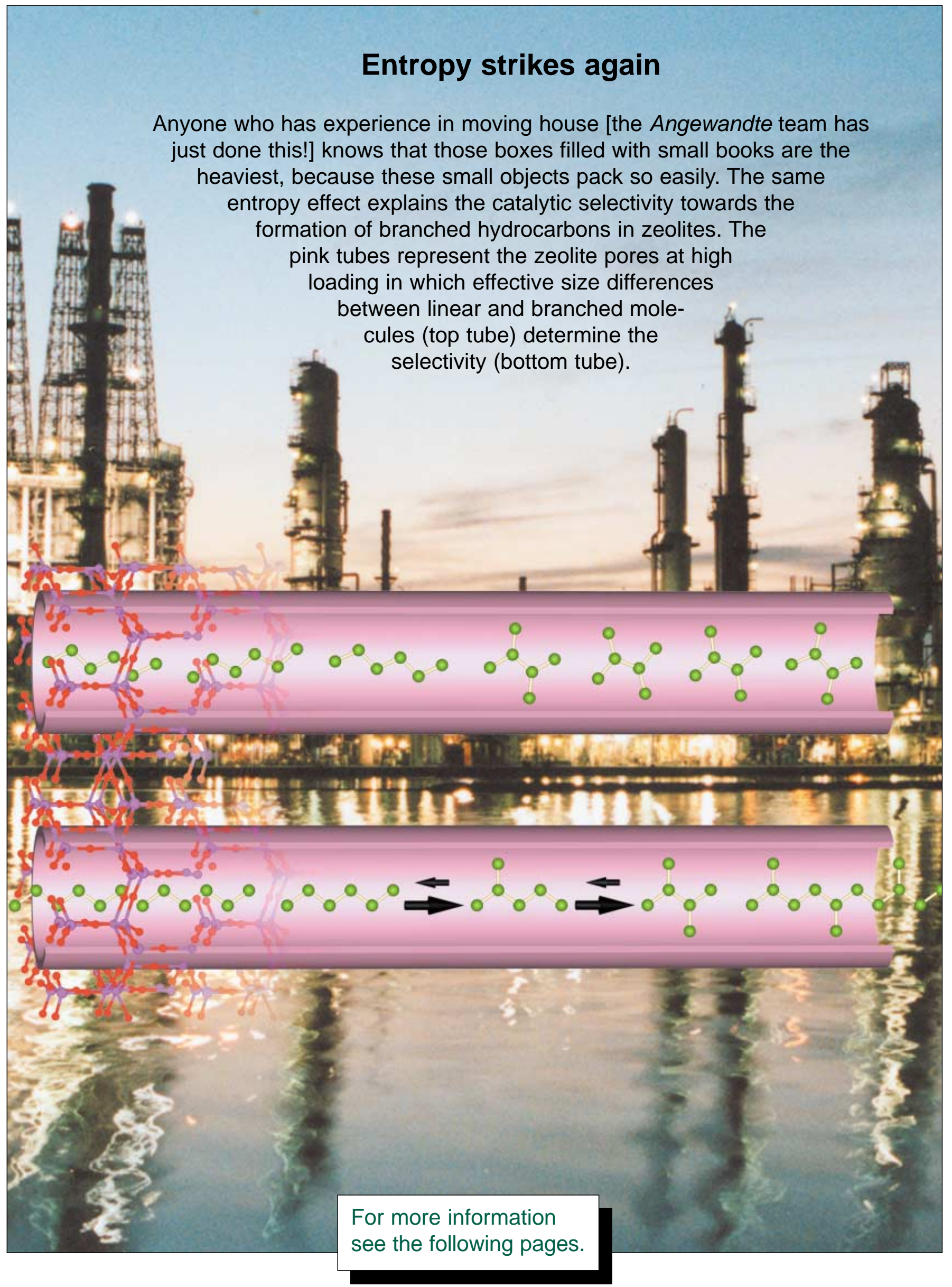




\section{Understanding Zeolite Catalysis: Inverse Shape Selectivity Revised**}

Merijn Schenk, Sofia Calero, Theo L. M. Maesen,

Lucas L. van Benthem, Martijn G. Verbeek, and

Berend Smit*

The molecular aspect of shape selectivity in zeolites is one of the key factors in zeolite science and technology of which a better understanding is required. The prediction of even qualitative aspects of a product distribution for a given reaction in a particular zeolite would be a significant step forward. ${ }^{[1]}$ In this respect the work of Santilli et al., ${ }^{[2]}$ which introduced the concept of inverse shape selectivity, can be seen as an important step. Inverse shape selectivity was used to explain the high yield of dibranched alkanes in AFI-type of zeolites. This concept is one of the first, apparently successful attempts, to predict some aspects of a product distribution by using molecular simulations. Traditional shape selectivity states, for example, that molecules will not (trans-)form if they are too bulky to fit inside a channel of a zeolite. Therefore, selectivity can be obtained by selecting zeolites with different channel sizes. Santilli et al. argued that the inverse process could also result in shape selectivity: those molecules form that have an optimal fit within the channels. For example, a difference in combustion properties (octane value) provides an economic incentive to prefer the formation of branched paraffins to linear paraffins. The concept of inverse shape selectivity tells us that this demand can be met by using zeolites that afford a maximum difference in Gibbs free energy between the branched and linear isomers. Using stateof-the-art simulation techniques and force fields, Santilli et al. demonstrated that their idea correlated perfectly with the measured selectivity. More importantly, Santilli et al.'s concept could be used to predict the optimal structure of a zeolite.

The initial motivation of our work was to redo and further refine the calculations of Santilli et al. by using more advanced simulation techniques and improved force fields. Whereas the simulations of Santilli et al. were limited to the energetic contributions, our approach, which uses the configurational-bias Monte Carlo (CBMC), allows computations of the entropic contributions as well. ${ }^{[3]}$ In addition, Santilli et al.

[*] Prof. B. Smit, Drs. M. Schenk, Dr. S. Calero, L. L. van Benthem, M. G. Verbeek

Department of Chemical Engineering

University of Amsterdam

Nieuwe Achtergracht 166, 1018 WV Amsterdam (The Netherlands)

Fax: (+31) 20-525-5604

E-mail: B.Smit@science.uva.nl

Dr. T. L. M. Maesen

ChevronTexaco

Energy Research and Technology

100 Chevron Way, Richmond, CA 94802-0627 (USA)

[**] These investigations are supported in part by the Netherlands Research Council for Chemical Sciences (CW) with financial aid from the Netherlands Technology Foundation (STW) and by the Netherlands Organization for Scientific Research (NWO) through PIONIER, and by the Stichting Nationale Computer Faciliteiten (National Computing Facilities Foundation) for the use of the supercomputer facilities. We thank the European Commission for a Marie Curie Individual Research Fellowship (to S.C.), and Christiaan Meijer for assistance with the artwork.

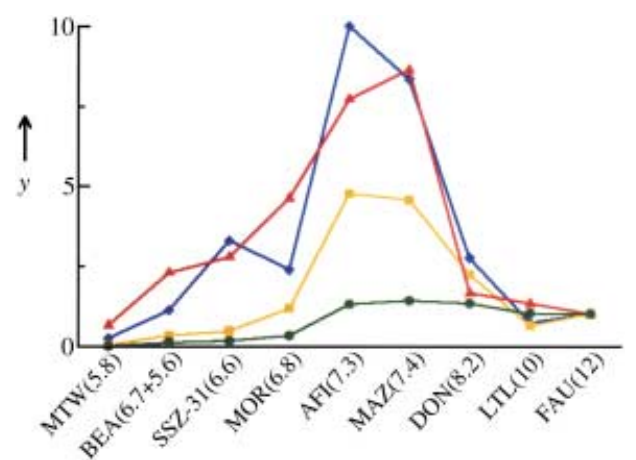

Figure 1. Experimental and simulated normalized $\mathrm{DMB} / n-\mathrm{C}_{6}$ yield ratios (y) for various zeolite structures at $T=577 \mathrm{~K}$ and $P=3000 \mathrm{kPa}$. The ratios were normalized by setting the value for the FAU-type zeolite at one. The experimental ratios (red) were determined from $n-\mathrm{C}_{16}$ hydroconversion experiments, ${ }^{[2]}$ the calculated ratios were taken from simulated adsorption isotherms of $2,2-\mathrm{DMB} / n-\mathrm{C}_{6}$ (yellow) and $2,3-\mathrm{DMB} / n-\mathrm{C}_{6}$ (blue) or from Henry coefficients (green). The numbers in parentheses are the average pore sizes $[\AA]$. DMB $=$ dimethylbutane, $n-\mathrm{C}_{6}=n$-hexane.

used the CVFF force field. Macedonia and Maginn have since shown that this force field is not particularly suited for simulating branched paraffins, ${ }^{[4]}$ for example, the CVFF force field does not reproduce the step that is clearly visible in the measured isotherms of isobutane adsorption by MFI-type silica at approximately half the loading. ${ }^{[2]}$ The force field we used does describe the adsorption isotherms of linear and branched alkanes in MFI quantitatively.

To our surprise, these "improved" simulations predict that the free energies of the linear and branched isomers in the "optimal" zeolites are nearly identical (see Figure 1). This result suggests that none of the "optimal" zeolites would favor the formation of branched alkanes. This prediction is in marked disagreement with the significant differences in selectivity observed experimentally. This situation suggests that the initial success of the predictions of Santilli et al. may have resulted from a fortuitous cancellation of errors. More importantly, our results also question the molecular interpretation of Santilli et al., that an optimum match between a branched molecule and the zeolite channel induces the selectivity. Here, we will demonstrate that the molecular basis of inverse shape selectivity is related to entropic effects inside the zeolite pores under conditions where the zeolites are (almost) fully saturated.

An important assumption in the calculations of Santilli et al. is that at reaction conditions the loading is sufficiently low, such that alkane - alkane interactions can be ignored. To test this assumption, we have computed adsorption isotherms for various $\mathrm{C}_{16}$ isomers, which is the reactant of the reaction of interest. Figure 2 shows a binary isotherm $(577 \mathrm{~K})$ of equal amounts of 2,5,8,11-tetramethyldodecane (a teM- $\mathrm{C}_{12}$ ) and $n$ - $\mathrm{C}_{16}$ in AFI-type pores. At low pressures there is little difference between the loading of the isomers, which is consistent with our observation that the free energies of these isomers at low loading are similar. However, if we consider the loading under reaction conditions $\left(3 \times 10^{3} \mathrm{kPa}\right)$, the adsorption isotherm shows that these pores are fully saturated with reactants. In addition, the AFI-type pores strongly prefer the adsorption of the shortest, most compact molecule, teM$\mathrm{C}_{12}$, to that of the longer molecule, $n-\mathrm{C}_{16}$. Similar simulations 


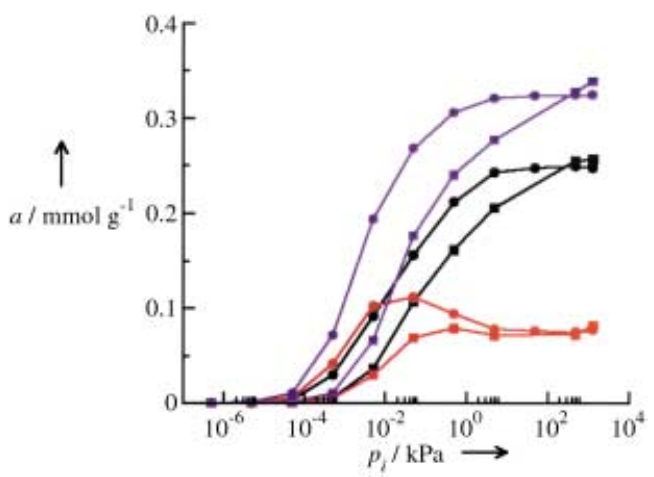

Figure 2. Simulated adsorption isotherms of equal amounts of teM-C

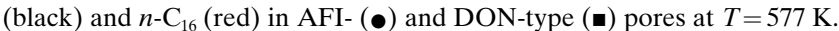
The total loading is given in purple. The grand canonical Monte Carlo simulations have been performed using the CBMC technique as described in Vlugt et al ${ }^{[9]} \mathrm{A}$ united atom force field has been used to describe the alkane-alkane interactions and the zeolite-alkane interactions. The parameters can be found in ref. [9]. AFI and DON have 1D linear pores with average channel diameters of 7.3 and $8.2 \AA$, respectively.

of different pore structures show that also the other systems are fully saturated with reactant under reaction conditions. These results clearly indicate that a key assumption underlying the mechanism of inverse shape selectivity does not hold. An alternative mechanism is therefore needed.

Since teM- $\mathrm{C}_{12}$ is the most likely source for dimethylbutane (DMB), and dimethyltetradecane $\left(\mathrm{dM}-\mathrm{C}_{14}\right)$ the most likely source for $n$-hexane $\left(n-\mathrm{C}_{6}\right),{ }^{[5,6]}$ it would be tempting to conclude that the $\mathrm{DMB} / n-\mathrm{C}_{6}$ ratio in the hydrocracking products is related to the differences in free energy of formation of these highly branched $\mathrm{C}_{16}$ isomers. However, Figure 2 also shows that similar differences in free energy are observed for the DON structure, which has a much larger pore diameter but does not yield a high $\mathrm{DMB} / n-\mathrm{C}_{6}$ ratio. ${ }^{[7]}$ These results demonstrate that at reaction conditions the zeolites are saturated with bulky $\mathrm{C}_{16}$ isomers, which have a very low diffusion coefficient, hence the exchange between gas phase and adsorbed phase will be extremely slow. Therefore we cannot assume that the adsorbed phase is in thermodynamic equilibrium with the gas phase. However, this does imply that the adsorbed phase will exhibit an intracrystalline thermodynamic equilibrium, that is, the zeolite will preferentially form those isomers that have the lowest free energy of formation at the conditions of interest. In other words, the product molecules are trapped sufficiently long in the zeolite molecules. that they will hydroisomerize towards their intracrystalline thermodynamic equilibrium distribution. If this assumption holds, the product distribution is determined by the relative intracrystalline Gibbs free energies of formation at reaction conditions. Since we do not know the composition of the various isomers at these conditions, we cannot compute these free energies exactly. The free energies can be approximated if we assume that the dominant factor is that the zeolite is completely saturated with hydrocarbons, and that the exact composition of the hydrocarbons is of secondary importance. In the structures of interest the molecules align nose to tail, and since the molecules only see the tail and nose of there neighbors it is of secondary importance whether the rest of the molecule is big or small.

We estimate the Gibbs free energy of formation of the hexane isomers by a binary mixture of 2,2- or 2,3-dimethylbutane (2,2-DMB or 2,3-DMB) and $n$-hexane $\left(n-\mathrm{C}_{6}\right)$ at reaction conditions and compute the relative free energies of these two components. In Figure 1 these DMB: $n-\mathrm{C}_{6}$ ratios are plotted for various zeolite structures. For small-pore zeolites the branched products are too bulky to form, giving selectivity towards the linear isomers. For the large-pore zeolites the linear and branched molecules are equally likely to form. An optimal selectivity is obtained for zeolites with pore size of approximately $7 \AA$. This observation is exactly what is found experimentally. ${ }^{[2]}$

A molecular explanation of our observation is shown in Figure 3. Talbot ${ }^{[8]}$ has shown that if we pack molecules in a 1D pore, at high pressures the smallest molecule will expel the bigger molecule. This is a purely entropic effect. A real zeolite is not a pure $1 \mathrm{D}$ system, therefore it is important to introduce
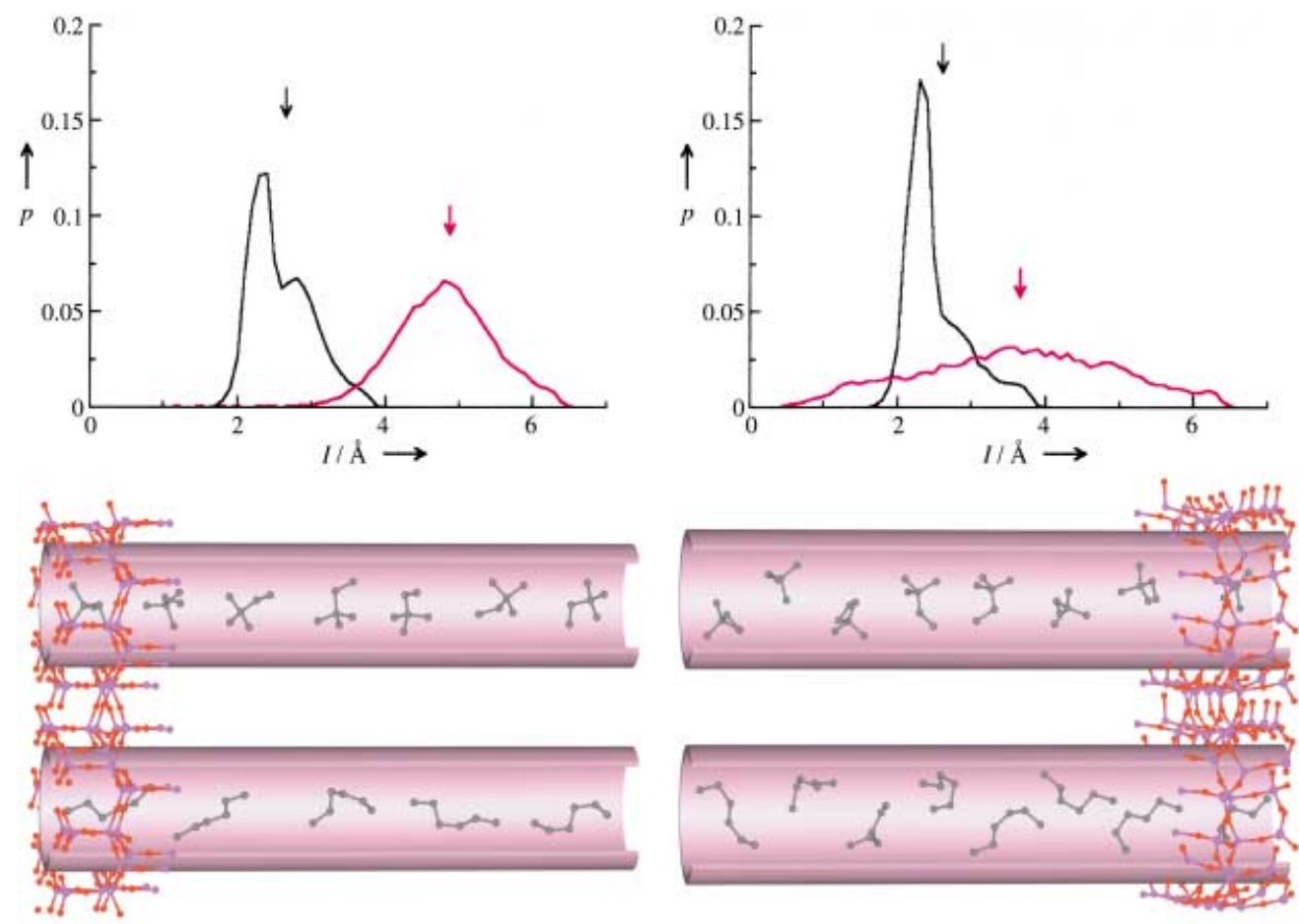

Figure 3. Adsorption of linear and branched hexane isomers in AFI (left) and AET (right). The "snapshots" show some typical conformations of the linear and branched isomers. In the smaller tube (AFI) the linear isomers are stretched while in the wider tube (AET) coiled conformations are also observed. Top: the projected end-toend distance distribution of $n$-hexane (red) and 2,2-DMB (black), the arrows indicate the effective size of the 
the effective size of a molecule, which is defined as the end-toend distance projected on the center line of the channel. Figure 3 shows the distribution of this distance for a large (AET) and optimal pore size (AFI). In the large-pore structure we observe for the linear isomer a very broad distribution, reflecting all possible conformations (curled and stretched) of this isomer. Hence, the effective size of the linear and branched isomer is nearly identical. If we reduce the size of the channel, this distribution is dominated by the stretched conformations, which increases the effective size of the linear isomers. Because the effective size of the linear isomer is large, these molecules are expelled at high pressure.

An important difference between the concept of inverse shape selectivity and our entropic explanation is the role of the zeolite. Inverse shape selectivity indicates that one should look at those zeolites, which have an optimal "match" for the branched isomer. In our mechanism the role of the zeolite is to provide an environment in which the length differences between the linear and branched isomers are maximum, which translates into an optimal pore diameter. For a given pressure, the maximum selectivity is determined by the relative effective sizes of the alkane molecules. The details of the channel structure are in this mechanism of secondary importance. This situation suggests that we can "optimize" any zeolite structure by tuning its diameter. In Figure 4 we have performed this optimization for several known zeolite structures by changing the pore diameters by a simple scaling factor. Of course, such an optimization cannot be performed in practice, but does illustrate our point that irrespective of the details of the zeolite a similar optimal selectivity is obtained for nearly identical channel dimensions. At lower temperatures or higher pressures the entropy effect is more pronounced and a better selectivity could be expected. The results shown in Figure 4 are at lower temperatures than those in Figure 1 ( $403 \mathrm{~K}$ versus $577 \mathrm{~K}$ ). The data at these lower temperatures give significantly higher selectivities. A similar effect can be expected from an increase of the pressure.

Two conclusions of practical importance can be drawn from our work. We have shown that there is a thermodynamic limit to the maximum selectivity that can be obtained for these types of reactions. This limit implies that any novel zeolite

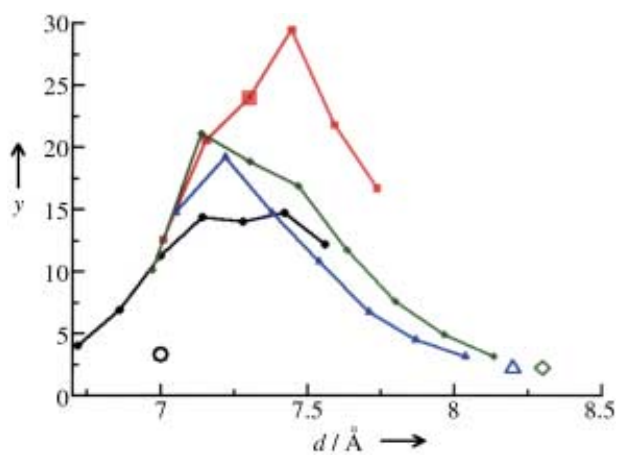

Figure 4. Normalized 2,2-DMB $/ n-\mathrm{C}_{6}$ yield ratios (with respect to the FAU selectivity) for some "optimized" pore structures at $T=403 \mathrm{~K}$ and $P=$ $1000 \mathrm{kPa}$. The size of MOR- (black, pore too small), AFI- (red, optimal), AET- (green, too wide), and DON-type (blue, too wide) channels was adjusted by scaling the coordinates. The open symbols represent the zeolite structures before resizing. MOR was first made circular before the scaling was applied. structures will have a selectivity towards branched paraffins which is at best similar to the selectivity of MAZ and AFI. A more successful approach to increase the selectivity would be to operate at higher pressure or lower temperatures, since the entropy effect is more pronounced at these conditions.

Received: February 1, 2002 [Z18631]

[1] J. Weitkamp, S. Ernst, L. Puppe in Catalysis and Zeolites (Eds.: J. Weitkamp, L. Puppe), Springer, Berlin, 1999, pp. 326-376.

[2] D. S. Santilli, T. V. Harris, S. I. Zones, Micropourous Mater. 1993, 1 , 329-341.

[3] D. Frenkel, B. Smit Understanding molecular simulations, 2nd ed., Academic Press, San Diego, 2002.

[4] M. D. Macedonia, E. J. Maginn, Mol. Phys. 1999, 96, 1375-1390.

[5] J. Weitkamp, S. Ernst, Catal. Today 1994, 19, 107-150.

[6] J. A. Martens, P. A. Jacobs in Zeolite Microporous Solids: Synthesis, Structure, and Reactivity (Eds.: E. G. Derouane, F. Lemos, C. Naccache, F. Ramão-Ribeiro), Kluwer, Amsterdam, 1992, pp. 511-529.

[7] M. Yoshikawa, P. Wagner, M. Lovallo, K. Tsuji, T. Takewaki, C.-Y. Chen, L. W. Beck, C. Jones, M. Tsapatsis, S. I. Zones, M. E. Davis, J. Phys. Chem. B 1998, 102, 7139-7147.

[8] J. Talbot, AIChE J. 1997, 43, 2471-2479.

[9] T. J. H. Vlugt, R. Krishna, B. Smit, J. Phys. Chem. B 1999, 103, 1102 1118.

\section{Reaction Principles and Crystal Structure Design for the Topochemical Polymerization of 1,3-Dienes}

Akikazu Matsumoto,* Kazuki Sada,* Kohji Tashiro,* Mikiji Miyata,* Takashi Tsubouchi, Toshihiro Tanaka, Toru Odani, Sadamu Nagahama, Tomoyuki Tanaka, Katsunari Inoue, Seishi Saragai, and Shinsuke Nakamoto

Crystal engineering is the planning of the properties and functions of crystalline materials by using preorganized molecules. ${ }^{[1]}$ This process involves the control of crystal

[*] Dr. A. Matsumoto, Dr. T. Tanaka, T. Odani

Department of Applied Chemistry

Graduate School of Engineering, Osaka City University and PRESTO-JST

Sugimoto, Sumiyoshi-ku, Osaka 558-8585 (Japan)

Fax: $(+81)$ 6-6605-2981

E-mail: matsumoto@a-chem.eng.osaka-cu.ac.jp

Dr. K. Sada, Prof. Dr. M. Miyata, T. Tanaka, K. Inoue

Department of Material and Life Science

Graduate School of Engineering, Osaka University

2-1 Yamadaoka, Suita, Osaka 565-0871 (Japan)

E-mail: sadatcm@mbox.nc.kyushu-u.ac.jp miyata@ap.chem.eng.osaka-u.ac.jp

Prof. Dr. K. Tashiro, Dr. S. Saragai, S. Nakamoto

Department of Macromolecular Science

Graduate School of Science, Osaka University

Toyonaka, Osaka 560-0043 (Japan)

E-mail: ktashiro@chem.sci.osaka-u.ac.jp

T. Tsubouchi, S. Nagahama

Department of Applied Chemistry

Graduate School of Engineering, Osaka City University Sugimoto, Sumiyoshi-ku, Osaka 558-8585 (Japan)

Supporting information for this article is available on the WWW under http://www.angewandte.org or from the author. 